\title{
Alternativas de Manejo guímico da Planta Daninha Digitaria ciliaris RESISTENTE AOS HERBICIDAS INIBIDORES DA ACCASE NA Cultura de SOJA ${ }^{1}$
}

\author{
Chemical Management Alternatives of the Weed Digitaria ciliaris Resistant to ACCASE \\ Inhibiting Herbicides in Soybean Crop
}

\author{
LÓPEZ-OVEJERO, R.F. ${ }^{2}$, PENCKOWSKI, L.H. ${ }^{3}$, PODOLAN, M.J. ${ }^{3}$, CARVALHO, S.J.P. ${ }^{4}$ e \\ CHRISTOFFOLETI, P.J. ${ }^{5}$
}

\begin{abstract}
RESUMO - Com o objetivo de avaliar a eficácia dos herbicidas inibidores da ACCase sobre uma população de capim-colchão (Digitaria ciliaris) com histórico de falha de controle, bem como propor herbicidas alternativos a serem aplicados em condições de pré e pós-emergência na cultura de soja, foram conduzidos dois experimentos a campo no município de PalmeiraPR, durante o ano agrícola 2003/2004. No primeiro experimento, foi avaliada a eficácia dos tratamentos envolvendo herbicidas com mecanismo de ação de inibição da ACCase ( $\mathrm{g} \mathrm{ha}^{-1}$ ): sethoxydim (230), clethodim (108), butroxydim (75), tepraloxydim (100), fluazifop-p-butil $(187,5)$, haloxyfop-r (60), propaquizafop (125), cyhalofop-butyl (225), fenoxaprop-p-ethyl + clethodim $(50+50)$, além de testemunha sem herbicidas. No segundo experimento, os tratamentos constaram de herbicidas com mecanismos de ação alternativos, visando também o teste de eficácia de controle da planta daninha $\left(\mathrm{g} \mathrm{ha}^{-1}\right)$ : trifluralina (2.700), clomazone (1.000), s-metolachlor (1.920), sulfentrazone (600), trifluralina + sulfentrazone $(2.100+400)$, clomazone + sulfentrazone $(600+400)$, S-metolachlor + sulfentrazone $(768+400)$ aplicados em condições de pré-emergência da planta daninha e da cultura e testemunha sem controle de plantas daninhas; os tratamentos apresentavam-se com ou sem complementação de controle através do herbicida imazethapyr (100), aplicado em condições de pós-emergência, com as plantas daninhas no estádio de duas a quatro folhas. Os resultados sugerem que a população estudada é resistente aos herbicidas inibidores da ACCase; os melhores resultados de eficácia de controle com os inibidores da ACCase foram obtidos com os herbicidas tepraloxydim, clethodim e butroxydim; os tratamentos com sulfentrazone isoladamente ou em mistura e os tratamentos com trifluralina, clomazone e S-metolachlor, em complementação com imazethapyr e imazethapyr isolado, foram eficazes no controle do biótipo resistente de Digitaria ciliaris, permitindo assim a recomendação destes tratamentos como alternativas de manejo de populações resistentes da planta daninha.
\end{abstract}

Palavras-chave: capim-colchão, resistência cruzada, biótipos, manejo.

\begin{abstract}
The objective of this work was to evaluate the efficacy of aplying ACCase inhibiting herbicides on a population of the weed large crabgrass (Digitaria ciliaris) with a history of control failure, as well as to propose alternative herbicides to be sprayed in pre-and post-emergence on the soybean crop. Thus, two field experiments were carried out in Palmeira (PR), Brazil, during the growing season 2003/2004. The first experiment evaluated the efficacy of the ACCase

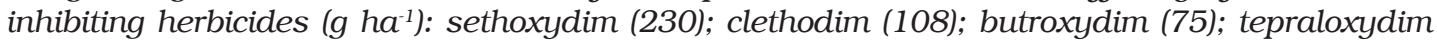
(100); fluazifop-p-butyl (187.5); haloxyfop-r (60); propaquizafop (125); cyhalofop-butyl (225); fenoxaprop-p-ethyl + clethodim $(50+50)$ and weeded check. The second experiment consisted of treatments using herbicides with alternative mechanism of action $\left(g \mathrm{ha}^{-1}\right)$ : trifluralina $(2,700)$;
\end{abstract}

1 Recebido para publicação em 17.10.2005 e na forma revisada em 5.5.2006.

Parte da tese do primeiro autor apresentada à ESALQ para obtenção do título de Doutor em Agronomia.

2 Eng.-Agr.., Doutorando em Fitotecnia na Escola Superior de Agricultura "Luiz de Queiroz" - ESALQ/USP, Caixa Postal 09, 13419-900 Piracicaba-SP, <rfloveje @ esalq.usp.br>. ${ }^{3}$ Eng.-Agr., Fundação ABC, Castro-PR, <luishenrique@ fundacaoabc.org.br>, <mario@fundacaoabc.org.br>. ${ }^{4}$ Eng.-Agr., Mestrando em Fitotecnia na ESALQ/USP, <sjpcarvalho@ yahoo.com.br>; ${ }^{5}$ Professor Associado do Departamento de Produção Vegetal da ESALQ/USP, <pjchrist@esalq.usp.br>

Planta Daninha, Viçosa-MG, v. 24, n. 2, p. 407-414, 2006 
clomazone (1,000); S-metolachlor (1,920); sulfentrazone (600); trifluralina + sulfentrazone (2,100 + 400); clomazone + sulfentrazone $(600+400)$; S-metolachlor + sulfentrazone $(768+400)$ in preemergence and a weeded check; all herbicides were applied with or without imazethapyr (100) application in post-emergence, sprayed when the weeds were at the stage of 2 to 4 leaves. The results suggested that the population studied is resistant to ACCase inhibiting herbicides; the best results of control efficacy with the ACCase inhibiting herbicides were obtained with tepraloxydim, clethodim and butroxydim; the treatments with sulfentrazone, alone or in mixture; the treatments with trifluralin, clomazone and S-metoalachlor, with imazethapyr complementation and imazethapyr alone were effective in controlling the resistant biotype of Digitaria ciliaris, showing that these treatments are management alternatives for the control of weed resistant populations.

Keywords: crabgrass, cross-resistance, biotypes, management.

\section{INTRODUÇÃO}

Uma das principais discussões atuais acerca do manejo de plantas daninhas nas culturas agrícolas brasileiras ou mundiais é o crescente aparecimento de novos casos de resistência a herbicidas. A extensão das áreas agrícolas atualmente detectada com a presença de biótipos resistentes de plantas daninhas pode ser considerada de pequena escala quando comparada com a área agrícola total, mas tem aumentado em uma taxa elevada. Os registros mundiais enumeram 296 biótipos resistentes, que abrangem 178 espécies (107 dicotiledôneas e 71 monocotiledôneas) em mais de 270.000 áreas agrícolas (HRAC, 2005).

A resistência de plantas daninhas aos herbicidas é definida como a capacidade natural e herdável de determinados biótipos, dentro de uma população, de sobreviver e se reproduzir após a exposição às doses que seriam letais aos indivíduos normais (suscetiveis) da mesma espécie. Denomina-se resistência cruzada os casos que envolvem diferentes herbicidas com mesmo sítio e/ou mecanismo de ação. Geralmente a resistência cruzada é conseqüência de uma alteração no sítio de ação enzimático do herbicida. A resistência múltipla, por sua vez, envolve herbicidas não relacionados quimicamente entre si e que apresentam mecanismos de ação diferenciados. Geralmente essa resistência é decorrente de processos metabólicos (Christoffoleti \& López-Ovejero, 2004).

Quando ocorrem plantas daninhas resistentes aos herbicidas em uma área, com densidade suficiente para limitar a produção das culturas agrícolas, tem-se a necessidade de mudanças nas práticas de manejo utilizadas. Assim, o estudo de alternativas de controle é fundamental para o adequado manejo dos biótipos resistentes (López-Ovejero et al., 2004). Para Boerboom (1999), o aspecto mais importante na prevenção e manejo da resistência é a recomendação de práticas e sistemas de produção onde a pressão de seleção de biótipos resistentes a determinado herbicida seja reduzida.

Segundo Peterson (1999), a mudança mais comum, adotada pelos agricultores em áreas onde foram detectados biótipos de plantas daninhas resistentes, é a adição de herbicidas alternativos, aplicados de forma isolada ou misturados em tanque com aqueles herbicidas para os quais foi detectada a resistência. No entanto, para Powles \& Holtum (1994), a alternativa de mistura de herbicidas no tanque de pulverização, bem como o uso de misturas formuladas ou aplicações seqüenciais de herbicidas para manejo e prevenção da resistência, está baseada no fato de que os ingredientes ativos controlam eficientemente os dois biótipos da mesma espécie, ou seja, o biótipo resistente a um dos herbicidas é controlado pelo outro ingrediente ativo da mistura. Peterson (1999) ressalta, também, que a troca do herbicida só se torna viável se existirem herbicidas alternativos que promovam o controle similar das plantas daninhas a custo compatível com o sistema de produção.

No Brasil, entre os casos mais freqüentes de resistência de plantas daninhas a herbicidas estão relacionados os inibidores das enzimas Acetolactato Sintase (ALS) e Acetil Co-A 
Carboxilase (ACCase), sobretudo na cultura da soja (Christoffoleti \& López-Ovejero, 2004). Especificamente para os herbicidas inibidores da ACCase, até o momento, foram identificados biótipos de Brachiaria plantaginea (capimmarmelada) e Digitaria ciliaris (capim-colchão) com comprovada resistência a esse mecanismo de ação (Gazziero et al.,1997; Cortez et al., 2002). Os biótipos resistentes a esse grupo de herbicidas podem surgir após seis a dez anos de pressão de seleção (Devine, 1997).

Para B. plantaginea são encontrados alguns trabalhos que propõem herbicidas alternativos para o manejo da espécie (Christoffoleti et al., 2001; López-Ovejero \& Christoffoleti, 2004), contudo existem poucas informações disponíveis sobre $D$. ciliaris. Assim, este trabalho foi conduzido com o objetivo de avaliar a eficácia dos herbicidas inibidores da ACCase sobre uma população de capim-colchão (Digitaria ciliaris), bem como propor herbicidas alternativos a serem aplicados em condições de pré e pós-emergência na cultura de soja.

\section{MATERIAL E MÉTODOS}

Dois experimentos foram conduzidos em área mantida sob sistema de semeadura direta sobre a palhada de aveia-preta (Avena strigosa), no município de Palmeira, PR, na safra de 2003/2004. A área apresentava histórico de aplicação de herbicidas inibidores da ACCase e relatos de falhas de controle da planta daninha Digitaria ciliaris. Para a dessecação foram realizadas duas aplicações de glyphosate, nas doses de 720 e $360 \mathrm{~g} \mathrm{ha}^{-1}$, aos 20 e aos dois dias antes da semeadura, respectivamente. A soja foi semeada em 31/10/2003, utilizando o cultivar "Aviara". As parcelas foram constituídas de sete linhas da cultura, espaçadas de 0,4 m, com 10,0 m de comprimento, o que corresponde a uma área total de $28 \mathrm{~m}^{2}$. A adubação de semeadura foi administrada com base na análise do solo (Tabela 1), utilizando $300 \mathrm{~kg} \mathrm{ha}^{-1}$ da fórmula 00-20-20.

Os tratamentos herbicidas utilizados foram selecionados a partir das recomendações disponíveis para controle do biótipo suscetivel de Digitaria ciliaris ou na freqüência de uso da molécula na cultura da soja, o que inclui herbicidas com mecanismo de ação do tipo inibidor da ACCase e outros mecanismos alternativos. Os tratamentos utilizados no primeiro e segundo experimentos são apresentados nas Tabelas 2 e 3, respectivamente. As doses dos herbicidas aplicados em pré-emergência foram escolhidas a partir das características físicoquímicas do solo (Tabela 1 - textura e matéria orgânica consideradas médias).

O delineamento experimental utilizado no primeiro experimento foi o de blocos ao acaso, com 10 tratamentos e 4 repetições. Para cada herbicida foi adicionado adjuvante recomendado pelo fabricante na concentração de $0,5 \% \mathrm{v} / \mathrm{v}$. A aplicação foi realizada quando as plantas daninhas apresentavam estádios de duas folhas a um perfilho e densidade média de infestação de 250 plantas $\mathrm{m}^{-2}$. O delineamento experimental utilizado no segundo experimento foi o de blocos ao acaso, em esquema de parcelas subdivididas, totalizando 16 tratamentos e 4 repetições. No âmbito das parcelas considerou-se o herbicida pré-emergente e, no das subparcelas, a presença ou ausência de complementação com imazethapyr. A aplicação em pré-emergência foi feita no dia da semeadura, sobre $2,3 \mathrm{t} \mathrm{ha}^{-1}$ de palha de aveia-preta. A aplicação do imazethapyr em pós-emergência foi realizada quando as plantas daninhas apresentavam de 2 a 4 folhas e densidade de 200 plantas $\mathrm{m}^{-2}$ na testemunha. O manejo de plantas daninhas de folhas largas, nos dois experimentos, foi realizado por meio da aplicação dos herbicidas lactofen (84 $\mathrm{g} \mathrm{ha}^{-1}$ ) e bentazon ( $480 \mathrm{~g} \mathrm{ha}^{-1}$ ), quando a cultura apresentava dois trifólios.

Tabela 1 - Características físico-químicas do solo. Palmeira-PR, 2003

\begin{tabular}{|c|c|c|c|c|c|c|c|c|c|c|c|}
\hline M.O. & $\mathrm{pH}$ & $\mathrm{P}_{\text {resina }}$ & $\mathrm{K}^{+}$ & $\mathrm{Ca}^{++}$ & $\mathrm{Mg}^{++}$ & $\left(\mathrm{H}^{+}+\mathrm{Al}^{+3}\right)$ & $\mathrm{CTC}$ & $\mathrm{V}$ & Argila & Silte & Areia \\
\hline$\left(\mathrm{g} \mathrm{dm}^{-3}\right)$ & $\left(\mathrm{CaCl}_{2}\right)$ & $\left(\mathrm{mg} \mathrm{dm}^{-3}\right)$ & \multicolumn{6}{|c|}{$\left(\mathrm{mmol}_{\mathrm{c}} \mathrm{dm}^{-3}\right)$} & \multicolumn{4}{c|}{$(\%)$} & \multicolumn{3}{|c|}{$\left(\mathrm{g} \mathrm{kg}^{-1}\right)$} \\
\hline 47 & 5,4 & 12 & 3,2 & 47 & 19 & 38 & 107,2 & 65 & 262 & 198 & 540 \\
\hline
\end{tabular}


Tabela 2 - Tratamentos utilizados no primeiro experimento para controle de Digitaria ciliaris supostamente resistente a herbicidas inibidores da ACCase. Palmeira-PR, 2003

\begin{tabular}{|l|c|c|}
\hline \multicolumn{1}{|c|}{ Herbicida } & \multicolumn{2}{|c|}{ Dose aplicada } \\
\hline Nome comum & g i.a. ha ${ }^{-1} \underline{1}$ l & $\begin{array}{c}\text { L ou kg de } \\
\text { p.c. ha }{ }^{-1} \underline{l}\end{array}$ \\
\hline - Cicloexanodionas & & \\
\hline 1. sethoxydim & 230,0 & 1,25 \\
\hline 2. clethodim & 108,0 & 0,45 \\
\hline 3. butroxydim & 75,0 & 0,30 \\
\hline 4. tepraloxydim & 100,0 & 0,50 \\
\hline - Ariloxifenoxipropiônicos & & \\
\hline 5. fluazifop-p-butil & 187,5 & 1,50 \\
\hline 6. haloxyfop-r & 60,0 & 0,50 \\
\hline 7. propaquizafop & 125,0 & 1,25 \\
\hline 8. cyhalofop-butyl & 225,0 & 1,25 \\
\hline 9. fenoxaprop-p-ethyl + clethodim & $50+50$ & 1,00 \\
\hline 10. Testemunha sem capina & - & - \\
\hline
\end{tabular}

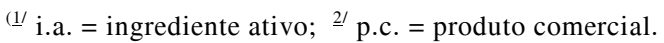

Os tratamentos herbicidas foram aplicados com pulverizador costal, à pressão constante $\left(\mathrm{CO}_{2}\right)$, equipado com barra de quatro pontas de pulverização do tipo "leque" XR 110.02 VS, espaçadas de $0,5 \mathrm{~m}$, com pressão de $23 \mathrm{lb} \mathrm{pol}^{-2}$ e volume da calda correspondente a $130 \mathrm{~L} \mathrm{ha}^{-1}$. A data de aplicação, o estádio fenológico da soja e as condições ambientais nos diferentes dias de aplicação estão especificados na Tabela 4.

As avaliações de eficácia de controle no primeiro experimento foram realizadas aos 14 e 28 DAP (Dias Após Aplicação Pós-emergente) e, no segundo, aos 20, 32 e 46 DAE (Dias Após Aplicação Pré-emergente). As avaliações de controle das plantas daninhas foram efetuadas empregando-se escala visual, considerando-se $0 \%$ para ausência de danos e 100\% para controle total (morte das plantas), comparados à testemunha sem herbicida (ALAM, 1974). No primeiro experimento também foi avaliada a massa fresca (MF) aos 28 DAP. A MF de Digitaria ciliaris foi obtida mediante o corte da parte aérea das plantas presentes na área de um gabarito de $0,25 \times 0,25 \mathrm{~m}\left(0,0625 \mathrm{~m}^{2}\right)$, lançado aleatoriamente duas vezes dentro das parcelas.

A análise dos dados foi feita por meio da aplicação do teste F sobre a análise de variância, a fim de verificar a existência de diferenças significativas entre os tratamentos. No primeiro experimento as médias foram comparadas pelo teste de Tukey em nível de $5 \%$ de probabilidade e, no segundo, a comparação das médias foi feita pelo teste $\mathrm{t}$ a $5 \%$ de probabilidade.

Tabela 3 - Tratamentos utilizados no segundo experimento para controle de Digitaria ciliaris supostamente resistente a herbicidas inibidores da ACCase. Palmeira-PR, 2003

\begin{tabular}{|c|c|c|c|}
\hline \multirow{2}{*}{$\begin{array}{c}\text { Tratamento } \\
\text { Pré-emergência }\end{array}$} & \multirow{2}{*}{ Pós-emergência } & \multicolumn{2}{|c|}{ Dose aplicada } \\
\hline & & g i.a. ha ${ }^{-1} \underline{1}^{\prime}$ & L p.c. ha ${ }^{-1} \stackrel{2}{\prime}$ \\
\hline \multirow{2}{*}{ 1. trifluralina } & - & 2700 & 4,5 \\
\hline & imazethapyr & $2.700+100$ & $4,5-1,0$ \\
\hline \multirow{2}{*}{ 2. clomazone } & - & 1000 & 2,0 \\
\hline & imazethapyr & $1.000+100$ & $2,0-1,0$ \\
\hline \multirow{2}{*}{ 3. S-metolachlor } & - & 1920 & 2,0 \\
\hline & imazethapyr & $1.920+100$ & $2,0-1,0$ \\
\hline \multirow{2}{*}{ 4. sulfentrazone } & $-\quad$ & 600 & 1,2 \\
\hline & imazethapyr & $600+100$ & $1,2-1,0$ \\
\hline \multirow{2}{*}{ 5. trifluralina + sulfentrazone } & - & $2.100+400$ & $3,5+0,8$ \\
\hline & imazethapyr & $2.100+400+100$ & $3,5+0,8-1,0$ \\
\hline \multirow{2}{*}{ 6. clomazone + sulfentrazone } & - & $600+400$ & $1,2+0,8$ \\
\hline & imazethapyr & $600+400+100$ & $1,2+0,8-1,0$ \\
\hline \multirow{2}{*}{ 7. S-metolachlor + sulfentrazone } & - & $768+400$ & $0,8+0,8$ \\
\hline & imazethapyr & $768+400+100$ & $0,8+0,8-1,0$ \\
\hline \multirow{2}{*}{ 8. Testemunha sem capina } & - & - & - \\
\hline & imazethapyr & 100 & 1,0 \\
\hline
\end{tabular}

${ }^{(1 /}$ i.a. $=$ ingrediente ativo; $\stackrel{2}{2}^{\text {p.c. }}=$ produto comercial. 
Tabela 4 - Data de aplicação, estádio fenológico da soja e condições ambientais nos diferentes dias da aplicação. Palmeira-PR, 2003

\begin{tabular}{|c|c|c|c|c|c|c|c|}
\hline \multirow{2}{*}{$\begin{array}{c}\text { Data de } \\
\text { aplicação }\end{array}$} & \multirow{2}{*}{ Estádio fenológico } & \multicolumn{2}{|c|}{ Horário } & \multicolumn{2}{|c|}{ Temperatura ${ }^{1 /}\left({ }^{\mathrm{C}} \mathrm{C}\right)$} & \multicolumn{2}{c|}{$\mathrm{UR}^{2 /}(\%)$} \\
\cline { 3 - 8 } & & Início & Final & Início & Final & Início & Final \\
\hline $31 / 10$ & Pré-emergência & $11 \mathrm{~h} 10$ & $11 \mathrm{~h} 38$ & 22,4 & 22,7 & 60 & 60 \\
\hline $21 / 11$ & 2 trifólios & $12 \mathrm{~h} 15$ & $12 \mathrm{~h} 45$ & 26,9 & 26,5 & 37 & 46 \\
\hline $25 / 11$ & 3 trifólios & $10 \mathrm{~h} 10$ & $10 \mathrm{~h} 40$ & 24,5 & 25,5 & 70 & 66 \\
\hline
\end{tabular}

(1/ Temperatura; $\stackrel{2}{2}$ Umidade relativa.

\section{RESULTADOS E DISCUSSÃO}

\section{Herbicidas inibidores da ACCase}

Os herbicidas inibidores da ACCase são divididos em dois grupos químicos: os ariloxifenoxipropionatos (APP) e as cicloexanodionas (CHD) (Harwood, 1999), que, embora apresentem diferenças em suas estruturas químicas, possuem afinidade pelo mesmo sítio de ação na ACCase (Rendina et al., 1989). Assim, os valores de controle de Digitaria ciliaris foram separados em função dos grupos de herbicidas inibidores de ACCase. Aos 14 DAP, o controle foi satisfatório (acima de 80\%) somente para os herbicidas clethodim, butroxydim e tepraloxydim, pertencentes ao grupo das cicloexanodionas; os herbicidas pertencentes ao grupo dos ariloxifenoxipropionatos e também o sethoxydim apresentaram controles inferiores a $40 \%$, por vezes de até $0 \%$ (Tabela 5).

Aos 28 DAP, os herbicidas tepraloxydim e clethodim mostraram controle acima de $87 \%$, e o butroxydim, inferior a $80 \%$. Os demais produtos aplicados mantiveram-se inferiores a $18 \%$. O herbicida clethodim isolado a $108 \mathrm{~g} \mathrm{ha}^{-1}$ foi superior em eficácia à sua mistura com fenoxaprop-p-ethyl (50 $\mathrm{g} \mathrm{ha}^{-1}$ de clethodim), sugerindo que o controle obtido deve estar muito mais relacionado com a aplicação de clethodim, de forma que a redução em sua dose resultou em perda de eficácia. Os resultados de controle apresentaram boa correlação com os dados de massa fresca, em que, quanto maior o controle do tratamento herbicida, menor a massa fresca presente nas parcelas (Tabela 5).

Os dados obtidos sugerem que a população estudada é resistente aos herbicidas inibidores da ACCase, com maior resistência ao grupo dos ariloxifenoxipropionatos, visto que não havia problemas com a qualidade dos produtos utilizados, com as doses recomendadas e com a época ou eficiência da aplicação. Embora a resistência ainda necessite ser confirmada por meio da comparação direta do biótipo suspeito com um biótipo sabidamente suscetivel (Ryan, 1970), já existem trabalhos que corroboram essa hipótese (López-Ovejero et al., 2005).

No trabalho conduzido por López-Ovejero et al. (2005), cinco populações de capimcolchão (quatro supostamente resistentes e uma sabidamente suscetivel) foram submetidas às aplicações de fluazifop-p-butil, sethoxydim e tepraloxydim, segundo a metodologia de curvas de dose-resposta. Os autores identificaram as quatro populações com suspeita como sendo resistentes aos inibidores da

Tabela 5 - Controle aos 14 e 28 DAP e produção de massa fresca (MF) aos 28 DAP de Digitaria ciliaris supostamente resistente aos herbicidas inibidores da ACCase, no primeiro experimento. Palmeira-PR, 2003

\begin{tabular}{|l|c|c|c|}
\hline \multicolumn{1}{|c|}{ Tratamento } & \multicolumn{2}{|c|}{ Controle (\%) } & MF \\
\hline Nome comum & 14 DAP & 28 DAP & $\left(\mathrm{g} \mathrm{m}^{-2}\right)$ \\
\hline - Cicloexanodionas & & & \\
\hline 1. sethoxydim & $0,0 \mathrm{c}$ & $0,0 \mathrm{c}$ & $343,6 \mathrm{ab}$ \\
\hline 2. clethodim & $86,0 \mathrm{a}$ & $87,0 \mathrm{a}$ & $22,4 \mathrm{c}$ \\
\hline 3. butroxydim & $85,0 \mathrm{a}$ & $70,0 \mathrm{a}$ & $101,5 \mathrm{c}$ \\
\hline 4. tepraloxydim & $87,5 \mathrm{a}$ & $90,0 \mathrm{a}$ & $13,2 \mathrm{c}$ \\
\hline - Ariloxifenoxipropiônicos & & & \\
\hline 5. fluazifop-p-butil & $0,0 \mathrm{c}$ & $0,0 \mathrm{c}$ & $350,3 \mathrm{ab}$ \\
\hline 6. haloxyfop-r & $0,0 \mathrm{c}$ & $0,0 \mathrm{c}$ & $242,6 \mathrm{~b}$ \\
\hline 7. propaquizanafop & $10,0 \mathrm{c}$ & $7,0 \mathrm{c}$ & $359,7 \mathrm{ab}$ \\
\hline 8. cyhalofop-butyl & $0,0 \mathrm{c}$ & $0,0 \mathrm{c}$ & $307,1 \mathrm{ab}$ \\
\hline 9. fenoxaprop-p-ethyl + clethodim & $40,0 \mathrm{~b}$ & $18,0 \mathrm{~b}$ & $249,9 \mathrm{~b}$ \\
\hline 10. Testemunha sem capina & $0,0 \mathrm{c}$ & $0,0 \mathrm{c}$ & $387,9 \mathrm{a}$ \\
\hline
\end{tabular}

Médias seguidas de letras minúsculas iguais nas colunas não diferem estatisticamente entre si pelo teste de Tukey a 5\% de probabilidade. 
ACCase, com diferentes níveis de resistência cruzada aos herbicidas com este mecanismo de ação. Também neste trabalho, o herbicida tepraloxydim apresentou controles satisfatórios quando aplicado na dose recomendada; as diferenças de controle ocorreram em subdoses e foram significativas.

\section{Herbicidas alternativos}

Os herbicidas S-metolachlor e sulfentrazone isolados ou o sulfentrazone em mistura com trifluralina, clomazone e S-metolachlor, aos $20 \mathrm{DAE}$, foram eficazes no controle do biótipo de Digitaria ciliaris (>80\%). Aos 32 e $46 \mathrm{DAE}$, os tratamentos de pré-emergência com trifluralina, clomazone e S-metolachlor apresentaram significativo incremento de controle quando receberam complementação de imazethapyr em pós-emergência. Ainda, os tratamentos de sulfentrazone e suas misturas controlaram de forma eficiente a planta daninha alvo sem precisar de complementação (Tabela 6).

Embora a aplicação de imazethapyr a $100 \mathrm{~g} \mathrm{ha}^{-1}$, de forma isolada no estádio de $2 \mathrm{a}$ 4 folhas, tenha sido eficiente no controle do biótipo resistente de Digitaria ciliaris, este tratamento pode provocar elevada toxicidade em algumas variedades de soja. No cultivar utilizado não foram observados sintomas de intoxicação por nenhum tratamento aplicado em pré ou pós-emergência. Os resultados de controle estão em concordância com estudo conduzido por Wiederholt \& Stoltenberg (1995), em que o biótipo de Digitaria sanguinalis resistente a sethoxydim e fluazifop-p foi suscetivel a imazethapyr e linuron.

Sabe-se que as recomendações de dose dos herbicidas pré-emergentes devem ser baseadas na textura do solo (teor de argila) e do teor de matéria orgânica (Christoffoleti \& LópezOvejero, 2005). Ainda, em sistema de plantio direto, os herbicidas podem ser interceptados pela palha e não atingir o solo onde efetivamente atuam. Em trabalho conduzido por Rodrigues et al. (1999), o sulfentrazone aplicado sobre a palha atingiu o solo, sendo lixiviado inclusive para camadas superiores a $10 \mathrm{~cm}$ de profundidade; no entanto, os herbicidas S-metolachlor (Cobucci et al., 2004), clomazone (Rodrigues, 1993) e trifluralina (Rodrigues et al., 1998) apresentaram restrições de uso. Vale ressaltar que o herbicida sulfentrazone apresenta tolerância diferencial entre os cultivares comerciais de soja. Em trabalho conduzido por Arruda et al. (1999), por exemplo, o cultivar BR-16 apresentou baixa tolerância ao sulfentrazone, uma vez que o herbicida reduziu a área foliar, o acúmulo de massa seca, a altura da planta e o comprimento das raízes.

Assim, este trabalho permitiu concluir que: o biótipo de capim-colchão (Digitaria ciliaris) presente na área não foi adequadamente controlado pelos herbicidas inibidores da ACCase, sobretudo os do grupo químico dos

Tabela 6 - Controle aos 20, 32 e 46 DAE de Digitaria ciliaris supostamente resistente aos herbicidas inibidores da ACCase, no segundo experimento. Palmeira-PR, 2003

\begin{tabular}{|c|c|c|c|c|c|}
\hline \multirow{3}{*}{ Tratamento } & \multirow{3}{*}{$20 \mathrm{DAE}$} & \multirow{2}{*}{\multicolumn{2}{|c|}{$\frac{32 \mathrm{DAE}}{\text { imazethapyr }}$}} & \multirow{2}{*}{\multicolumn{2}{|c|}{$\begin{array}{c}46 \text { DAE } \\
\text { imazethapyr }\end{array}$}} \\
\hline & & & & & \\
\hline & & Sem & Com & Sem & Com \\
\hline 1. trifluralina & $50,0 \mathrm{~b}$ & $61,7 \mathrm{Bc}$ & $94,4 \mathrm{Aa}$ & $56,7 \mathrm{Bc}$ & $98,7 \mathrm{Aa}$ \\
\hline 2. clomazone & $79,0 \mathrm{ab}$ & $81,7 \mathrm{Bb}$ & $96,3 \mathrm{Aa}$ & $83,3 \mathrm{Bb}$ & $97,3 \mathrm{Aa}$ \\
\hline 3. S-metolachlor & $85,7 \mathrm{ab}$ & $80,0 \mathrm{Bb}$ & $96,3 \mathrm{Aa}$ & $83,3 \mathrm{Bb}$ & $97,3 \mathrm{Aa}$ \\
\hline 4. sulfentrazone & $100,0 \mathrm{a}$ & $95,7 \mathrm{Aa}$ & $98,3 \mathrm{Aa}$ & $97,3 \mathrm{Aa}$ & $99,0 \mathrm{Aa}$ \\
\hline 5. trifluralina + sulfentrazone & $99,0 \mathrm{a}$ & $99,3 \mathrm{Aa}$ & 99,7 Aa & $100,0 \mathrm{Aa}$ & $100,0 \mathrm{Aa}$ \\
\hline 6. clomazone + sulfentrazone & $97,3 \mathrm{a}$ & $91,7 \mathrm{Aa}$ & $100,0 \mathrm{Aa}$ & $99,3 \mathrm{Aa}$ & $99,3 \mathrm{Aa}$ \\
\hline 7. S-metolachlor + sulfentrazone & $100,0 \mathrm{a}$ & $96,7 \mathrm{Aa}$ & $100,0 \mathrm{Aa}$ & $99,0 \mathrm{Aa}$ & $100,0 \mathrm{Aa}$ \\
\hline 8. Testemunha sem capina & $0,0 \mathrm{c}$ & $0,0 \mathrm{Bd}$ & $88,3 \mathrm{Aa}$ & $0,0 \mathrm{Bd}$ & $93,0 \mathrm{Aa}$ \\
\hline
\end{tabular}

Médias seguidas de letras minúsculas iguais nas colunas e maiúsculas nas linhas não diferem estatisticamente entre si pelo teste $\mathrm{t}$ a $5 \%$ de probabilidade. 
ariloxifenoxipropionatos, fato que sugere a ocorrência de resistência; e, exceto trifluralina aplicada isoladamente, os herbicidas aplicados em pré-emergência, com ou sem complementação de imazethapyr em pós-emergência, podem ser utilizados no manejo de populações resistentes de Digitaria ciliaris, porém podem apresentar restrições de manejo quando comparados aos pós-emergentes.

\section{AGRADECIMENTOS}

Ao HRAC-BR, pelo suporte financeiro para a realização da pesquisa; e à Fundação $\mathrm{ABC}$, pela disponibilização das áreas de campo e pelo suporte técnico.

\section{LITERATURA CITADA}

ARRUDA, J. S.; LOPES, N. F.; BACARIN, M. A. Crescimento de plantas de soja em função de doses de sulfentrazone. Planta Daninha, v. 17, n. 3, p. 375-386, 1999.

ASOCIATION LATINO AMERICANA DE MALEZAS. Recomendaciones sobre unificación de los sistemas de evaluación en ensayos de control de malezas. ALAM, v. 1, p. 35-38, 1974.

BOERBOOM, C. M. Nonchemical options for delaying weed resistance to herbicides in Midwest cropping systems. Weed Technol., v. 13, p. 636-642, 1999.

CHRISTOFFOLETI, P. J.; LÓPEZ-OVEJERO, R. F. Dinâmica dos herbicidas aplicados ao solo na cultura da cana-de-açúcar. São Paulo: BASF, 2005. 49 p.

CHRISTOFFOLETI, P. J.; LÓPEZ-OVEJERO, R. F. Definições e situação da resistência de plantas daninhas aos herbicidas no Brasil e no mundo. In: CHRISTOFFOLETI, P. J. (Coord.). Aspectos de resistência de plantas daninhas a herbicidas. Londrina: Associação Brasileira de Ação a Resistência de Plantas aos Herbicidas (HRAC-BR), 2004. p. 3-22.

CHRISTOFFOLETI, P. J.; KEHDI, C. A.; CORTEZ, M. G. Manejo da planta daninha Brachiaria plantaginea resistente aos herbicidas inibidores da ACCase. Planta Daninha, v. 19, n. 1, p. 61-66, 2001.

COBUCCI, T. et al. Comportamento de herbicidas com efeito residual em diferentes coberturas na cultura do feijoeiro. Planta Daninha, v. 22, n. 4, p. 591-596, 2004.
CORTEZ, M. G.; MADUREIRA, A.; LÓPEZ-OVEJERO, R. Resistência de Digitaria sp. a herbicidas inibidores da acetil coenzima A carboxilase (ACCase). In: CONGRESSO BRASILEIRO DA CIÊNCIA DAS PLANTAS DANINHAS, 23., 2002, Londrina. Resumos... Londrina: SBCPD/ Embrapa Clima Temperado, 2002. p. 191.

DEVINE, M. D. Mechanisms of resistance to acetylcoenzime A carboxylase inhibitors: a review. Pestic. Sci., v. 51, p. 259-264, 1997.

GAZZIERO, D. L. P. et al. Resistência de biótipos de Brachiaria plantaginea aos herbicidas inibidores da ACCase aplicados em soja. In: CONGRESSO BRASILEIRO DA CIÊNCIA DAS PLANTAS DANINHAS, 21., 1997, Caxambu. Resumos... Caxambu: SBCPD, 1997. p. 88.

HARWOOD, J. L. Graminicides which inhibit lipid synthesis. Pest. Out., v. 10, p. 154-158, 1999.

HERBICIDE RESISTANCE ACTION COMMITTEE HRAC. Disponível em: <http://www.plantprotection.org/ hrac>. Acesso em: 23 abr. 2005.

LÓPEZ-OVEJERO, R. F. et al. Resistência de populações de capim-colchão (Digitaria ciliaris) aos herbicidas inibidores da acetil Co-A carboxilase. Planta Daninha, v. 23, n. 3, p. 543-549, 2005.

LÓPEZ-OVEJERO, R. F.; CHRISTOFFOLETI, P. J.; VARGAS, L. Resistência de plantas daninhas a herbicidas. In: VARGAS, L.; ROMAN, E. S. (Eds.). Manual de manejo e controle de plantas daninhas. Bento Gonçalves: Embrapa Uva e Vinho, 2004. p. 185-214.

LÓPEZ-OVEJERO, R. F.; CHRISTOFFOLETI, P. J. Recomendações para prevenção e manejo da resistência a herbicidas. In: CHRISTOFFOLETI, P. J. (Coord.). Aspectos da resistência de plantas daninhas a herbicidas. Londrina: Associação Brasileira de Ação a Resistência de Plantas aos Herbicidas (HRAC-BR), 2004. p. 49-86.

PETERSON, D. E. The impact of herbicide-resistant weeds on Kansas agriculture. Weed Technol., v. 13, p. 632-635, 1999.

POWLES, S. B.; HOLTUM, J. A. M. Herbicide resistance in plants: biology and biochemistry. Boca Raton: Lewis, 1994. $353 \mathrm{p}$.

RENDINA, A. R. et al. Kinetics of inhibition of acetyl-CoA carboxylase by the aryloxyphenoxypropionate and cyclohexanedione graminicides. In: THE BRIGHTON CROP PROTECTION CONFERENCE - WEEDS, 1989, Proceedings... Farnhan: British Crop Protection Council, 1989. p. 163-172.

RODRIGUES, B. N. et al. Influência da cobertura morta no comportamento do herbicida sulfentrazone. Planta Daninha, v. 17, n. 3 , p. $445-458,1999$. 
RODRIGUES, B. N. et al. Influência da cobertura morta no comportamento do herbicida trifluralin. Planta Daninha, v. 16, n. 2, p. 163-173, 1998.

RODRIGUES, B. N. Influência da cobertura morta no comportamento dos herbicidas imazaquin e clomazone. Planta Daninha, v. 11, p. 21-28, 1993.
RYAN, G. F. Resistance of common groundsel to simazine and atrazine. Weed Sci., v. 18, p. 614-620, 1970.

WIEDERHOLT, R. J.; STOLTENBERG, D. E. Cross-

Resistance of large crabgrass (Digitaria sanguinalis) accession to aryloxyphenoxypropionate and cyclohexanedione herbicides. Weed Technol., v. 9, p. 518-524, 1995. 